\title{
First Measurements with the LHCb Experiment
}

\author{
Markward Britsch on behalf of the $\mathrm{LHCb}$ collaboration \\ Max-Planck-Institut für Kernphysik \\ PO Box 103980, 69029 Heidelberg - Germany
}

\begin{abstract}
The LHCb detector covers the forward angular region complementary to the angular coverage by the general purpose LHC experiments. With the first data, measurements of inclusive charged particle production and $V^{0}$ production will be performed and compared to particle production models. Focusing on ratios, such as positives/negatives or lambda-bar/lambda, many systematic effects cancel and publishable results are expected soon after the turn-on of LHC. The talk will present the analysis strategies, the physics questions and the results expected from the first $10^{8}$ minimum bias $p p$-collisions at a center-of-mass energy of $10 \mathrm{TeV}$, which can be recorded within one day of stable running.
\end{abstract}

\section{Introduction}

a The baryon asymmetry of the universe indicates that there should be a source of CP violation beyond that from the Standard Model. Alternative models often predict the existence of new kinds of particles. One way to tackle this puzzle are precision measurements of $C P$ violation and rare decays which are in general sensitive to new kinds of heavy particles from loop diagrams. $\mathrm{LHCb}[2]$ is an experiment at the $p p$-collider LHC dedicated to these kinds of measurements. This means that we are mainly interested in heavy flavor physics. As most $b \bar{b}$-quark pairs are produced in forward and backward direction, LHCb was build as a forward spectrometer with a pseudorapidity coverage for $B$-mesons of $1.9<\eta<4.9$. This is complementary to the central $\eta$-coverage of ATLAS and CMS of about $-2.5<\eta<2.5$. In addition $\mathrm{LHCb}$ can measure down to lower transverse momenta.

To achieve its ambitious goals, the LHCb experiment has a good vertex resolution, dedicated triggers and various precise particle identification (PID) systems including two Ring Imaging Cherenkov counters (RICH). In the following we will concentrate on the tracking system which consists of the vertex detector, called Vertex Locator or VeLo, two tracking stations in front of the magnet (called TT stations) and the main tracker consisting of three stations behind the magnet. The main tracker is split into the inner and outer part. The inner tracker is much smaller than the outer tracker and covers the largest pseudorapidities where the track multiplicities are highest.

The conditions of the LHC in 2009/2010 will be different from the nominal ones with a lower center-of-mass energy of $\sqrt{s}=8-10 \mathrm{TeV}$ and a lower luminosity of up to $\mathcal{L} \sim$ $10^{32} \mathrm{~cm}^{-2} \mathrm{~s}^{-1}$. The nominal values for $\mathcal{L}$ and $\sqrt{s}$ are not the crucial parameters for the LHCb experiment and its full physics program can start in 2009/2010. The LHCb experiment's commissioning is well on the way using cosmic data as well as beam-gas and beam on collimator data from the LHC commissioning in fall 2008 [3].

First measurements will be done using events collected by a minimum bias trigger and the nominal data logging rate of $\mathrm{LHCb}$ of $2 \mathrm{kHz}$. With this we can expect to record $10^{8}$ events within the first few days after the start of LHC. Initially, we will only use tracking

${ }^{\mathrm{a}}$ For the slides of this talk see [1]. 
without PID as long as the PID system is not calibrated. The first goal will be to measure particle ratios like those for charged tracks, $K_{s}^{0}, \Lambda$ or $D$-mesons, i.e., particle to anti-particle ratios as well as $K_{s}^{0} / \Lambda$. Here most systematics cancel and no luminosity measurement is needed in contrast to cross section measurements. The studies discussed below are based on $9.5 \cdot 10^{6}$ LHCb Monte Carlo events produced in 2006 with $\sqrt{s}=14 \mathrm{TeV}$.

\section{Physics topics}

The physics topics covered here are inclusive production, strangeness production and charm signals, all shown to be feasible with the very first data. While interesting in their own right, they will serve as stepping stones to heavy flavor physics, especially to $B$-decays with $K_{s}^{0}$ as daughter, $b$-baryon spectroscopy and lifetimes, and radiative b-baryon decays. In addition they will be used as input for tuning the Monte Carlo event generator and used to test fragmentation models for multi particle production.

Elements of multi particle production are fragmentation, color (re)connection and multiple parton interaction (MPI) which will be especially important at the LHC. Color (re)connection deals with the multitude of possibilities to connect the differently colored lines of the quarks and gluons in the corresponding Feynman diagram to result in a color neutral state in the end. MPI means that there is more than one parton of each proton involved in the collision. In recent years new models have been developed, see, e.g., [5].

Strangeness is an additional probe for the fragmentation process, since the $s$-quark is still a comparatively light particle, it is mainly created in the hadronization stage. In addition some new models predict the beam baryon number to reach lower $\eta$ at low $p_{t}$ [5]. This can be seen in Figure 1 where the ratio of $\bar{\Lambda}$ to $\Lambda$ are plotted versus pseudorapidity for two old and two new models. In the central pseudorapidity range the models hardly differ while in

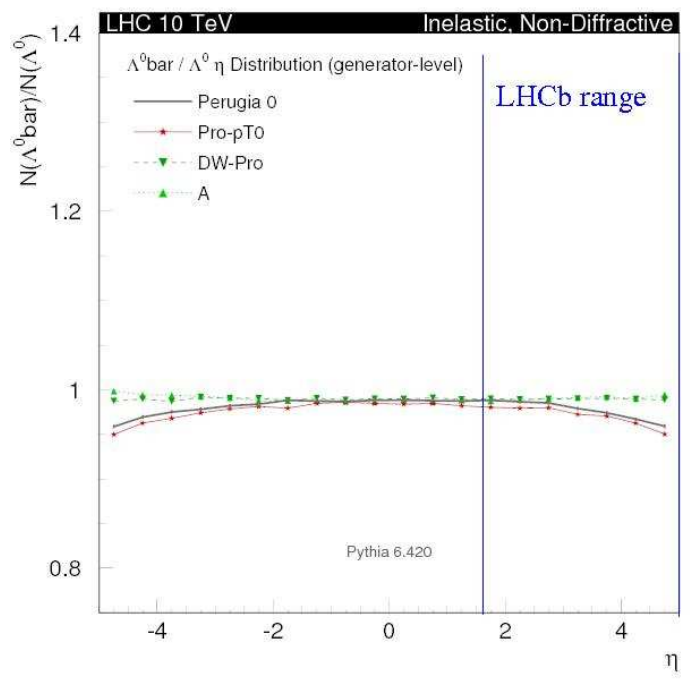

Figure 1: $\bar{\Lambda}$ to $\Lambda$ number ratio plotted versus pseudorapidity for old (triangles) and new (no symbol, star) models at LHC. The vertical lines mark the LHCb range. Taken from [4].

the range of $\mathrm{LHCb}$ indicated in the figure shows that the difference is up to $5 \%$.

\section{Inclusive production}

For the charged track ratios we generated one million minimum bias events and selected tracks in the range $100 \mathrm{MeV} / c<p_{t}<8000 \mathrm{MeV} / c$ and $1.8<\eta<5.1$. A minimal requirement for this measurement to be possible will be a working main tracker. These kind 

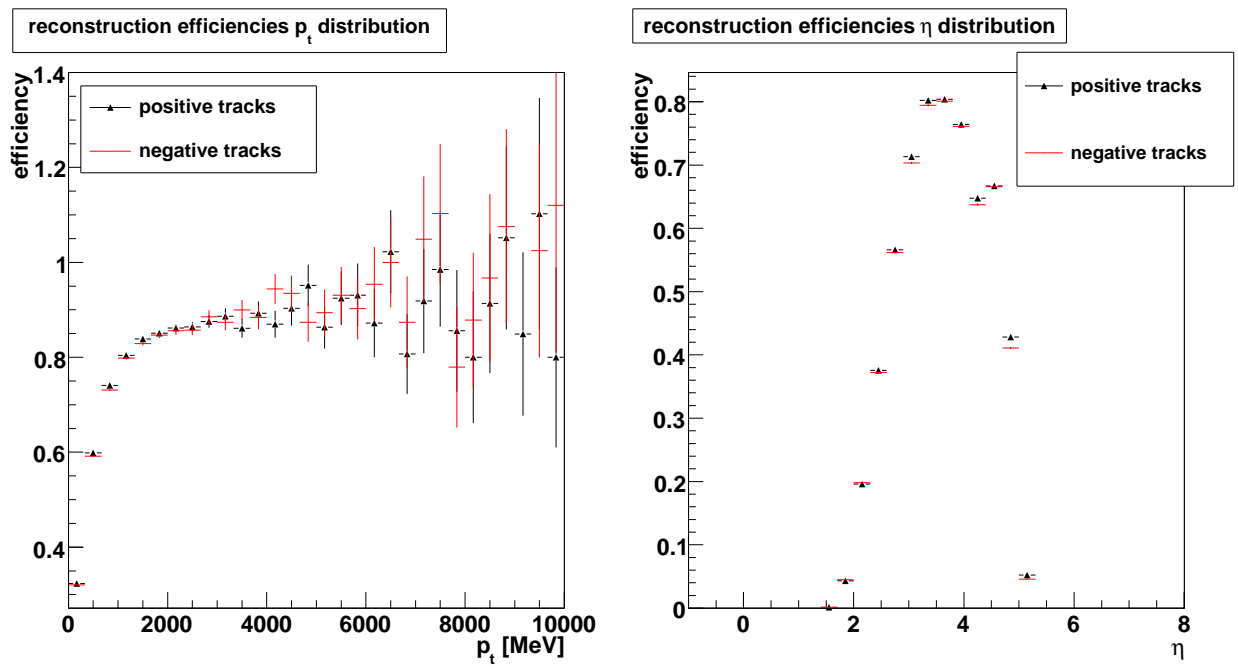

Figure 2: Reconstruction efficiencies for positive (black, triangles) and negative (red) tracks plotted versus transverse momentum (left) and pseudorapidity (right). For the $p_{t}$ distributions particles are integrated over $1.8<\eta<5.1$, for the $\eta$-distributions over $100 \mathrm{MeV} / c$ $<p_{t}<8000 \mathrm{MeV} / c$.

of studies will be vital for understanding charge asymmetries but will also be used for Monte Carlo tuning and comparison with different fragmentation models.

To compare the reconstructed tracks to the simulated ones we used the following selection criteria. For the generated tracks, only those from the events with exactly one primary vertex $(\mathrm{PV})$ have been accepted. Both elastic and diffractive events were rejected. Only long lived particles (lifetime $\tau>1 \mathrm{~ns}$ ) in our $\left(\eta, p_{t}\right)$-range coming from the PV or from a short lived particle from the PV have been selected. For the reconstructed tracks only events with exactly one PV have been used. The track selection required the tracks to be compatible with coming from the PV (i.e., impact parameter $<0.15 \mathrm{~mm}$ ) and to have hits in both VeLo and main tracker.

In Figure 2 the reconstruction efficiencies versus transverse momentum $\left(p_{t}\right)$ and pseudorapidity are shown. Figure 3 shows the charged track ratios for generated and reconstructed tracks as a function of $p_{t}$ and $\eta$. The differences between generated and reconstructed ratios are $\lesssim 5 \%$ for most of the bins. Still, it will be necessary to correct the reconstructed ratios for acceptance. If we use the correction factors obtained from the simulation, we need to trust the simulation result to a level of $20 \%$ in order to get a systematic error of $\sim 5 \% \times 20 \%$ $=1 \%$.

\section{Cross section ratios for identified particles}

For the strange particle selection we use the decays $K_{s}^{0} \rightarrow \pi^{+} \pi^{-}, \Lambda \rightarrow p \pi^{-}$and $\bar{\Lambda} \rightarrow$ $\bar{p} \pi^{+}$. Candidates are pairs of oppositely charged tracks. The selection considered in this study is based on the values of a reconstructed variables rather than their significances. 

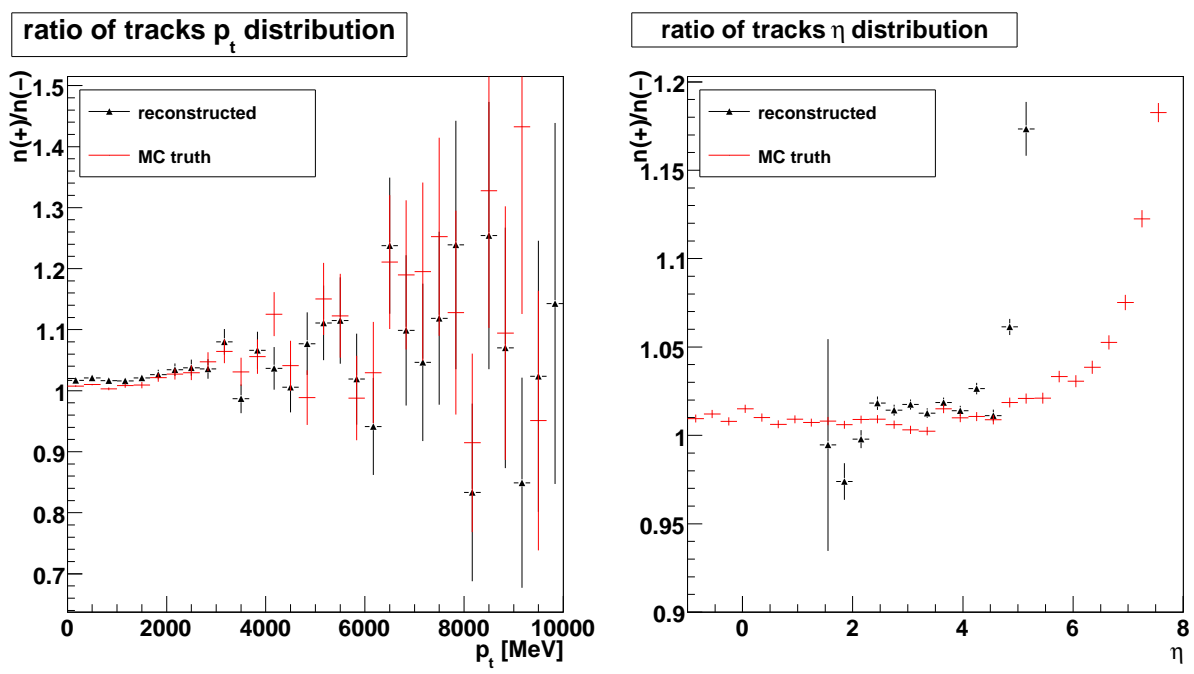

Figure 3: Number ratio of positive to negative tracks as reconstructed (black, triangles) and generated (red) plotted versus transverse momentum (left) and pseudorapidity (right).

Although the later will improve the sensitivity of the analysis, they will only be employed at a later stage since they require a more complete understanding of the detector. The studies are based on 9.5 million minimum bias events, i.e., an order of magnitude less than what we expect to have as the first data (see Section 1). Minimal requirements for these measurements will be a working VeLo and main tracker. It can be used to check momentum calibration and is important for RICH calibration.

Figure 4 shows the reconstructed ratios of $\bar{\Lambda}$ to $\Lambda$ as a function of pseudorapidity. $\Lambda$ and $\bar{\Lambda}$ were selected requiring that the distance of closest approach between the two tracks $\leq 0.3 \mathrm{~mm}$, flight-length $\gamma \beta$ $\geq 4 \mathrm{~mm}$, impact parameter with respect to the $\mathrm{PV}$ $\leq 0.1 \mathrm{~mm}$ and daughter transverse momentum with respect to the direction of flight of the mother particle $\geq 10 \mathrm{MeV} / c$. In this plot we see mostly a $\sim 4 \%$

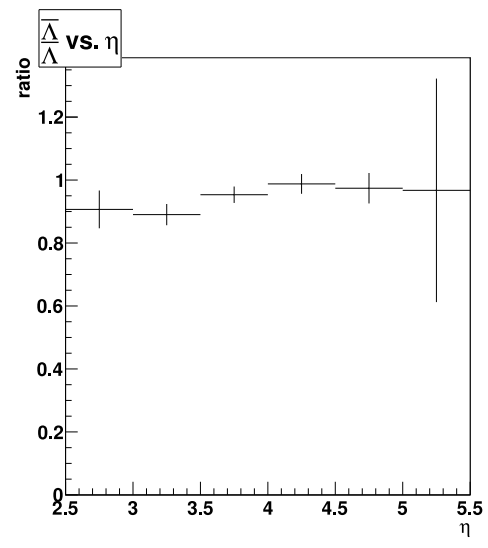

statistical error for the ratios. Extrapolating to the expected $10^{8}$ events gives an error of $1.3 \%$ which should be good enough to decide between new and old models (see Figure 1).

The $D$-meson selection is similar to that of the strange particles using the decays $D^{0} \rightarrow$ $K^{-} \pi^{+}, \bar{D}^{0} \rightarrow K^{+} \pi^{-}$and $D^{ \pm} \rightarrow K^{\mp} \pi^{ \pm} \pi^{ \pm}$. We use a cuts based selection and a multivariate analysis (MVA) 6]. MVA is just a more sophisticated method to cut on the phase space and thus still consistent with being conservative. Only geometric and kinematic variables (no significances and no PID) are used for the cuts and in the MVA. The Minimal requirements 

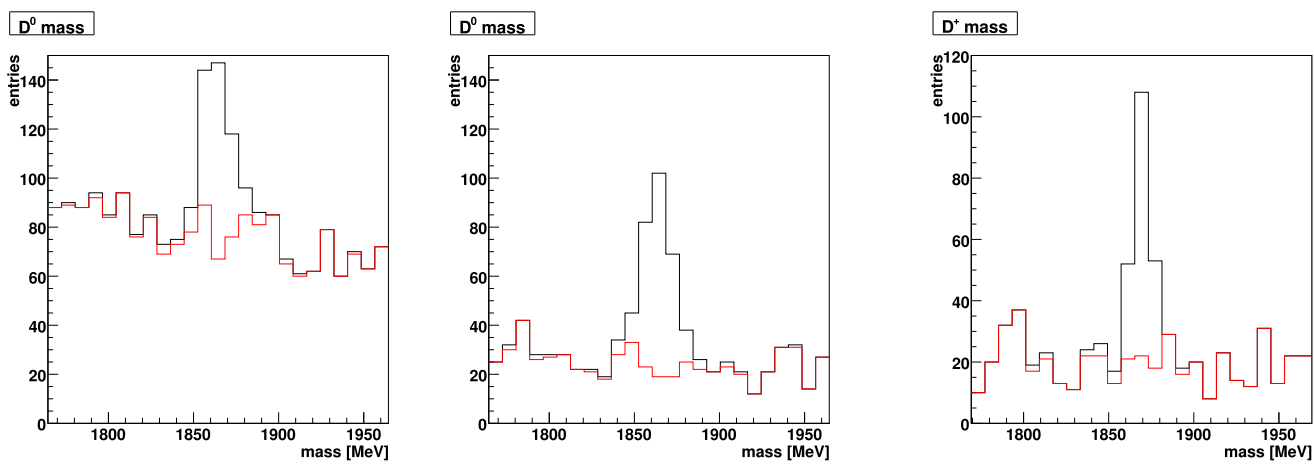

Figure 5: $D^{0}$ mass peak for the cuts based (left) and MVA (center) selection. The MVA parameters have been chosen in a way to get the same signal yield for comparison. On the right the $D^{+}$mass peak using the cuts based selection is shown.

for these measurements are a well aligned VeLo and main tracker.

In Figure 5 the mass peaks for the $D^{0}$ and $D^{+}$are shown. The MVA parameters have been chosen in a way to get the same signal yield as for the cuts based analysis. One can see by comparing the plots for the $D^{0}$ mass peaks that the MVA reduces the background by about a factor of three here. For the charged $D$-mesons we used the cuts based analysis only. The expected sensitivity on $D$ selection is about 2000 particles for each of the above charm species for $100 \mathrm{M}$ events. For $p_{t}<12 \mathrm{GeV}$, rapidity $1.8<y<4.5$ we expect an error on $\overline{D^{0}} / D^{0}$ cuts based of $7 \%$, MVA $5 \%$, for the $D^{-} / D^{+}$we expect to get $6 \%$.

\section{Conclusion and outlook}

With the first $10^{8}$ minimum bias events which corresponds to about one day of running we expect to measure charged track ratio distributions with $\sim 1 \%$ error and we expect to be able to probe fragmentation models by strange particle ratios. We expect to reconstruct $\sim 2000 D^{0 / \pm}$ and the open charm ratios with about $5 \%$ precision.

More detailed Monte Carlo studies are on the way. In addition we will look into other strange baryons $\left(\Xi^{-}, \Omega^{-}\right)$ratios and we will look for $b$-baryons like $\Lambda_{b}$ or $\Xi_{b}$. Of course corresponding cross section measurements are also planed.

\section{References}

[1] Slides: http://indico . cern . ch/contributionDisplay . py? contribId=258\&sessionId=3\& conf Id=53294.

[2] The LHCb Collaboration. The LHCb Detector at the LHC. Journal of Instrumentation, 8:8005-+, August 2008.

[3] de Capua for the LHCb collaboration. The LHCB Commissioning. In oai:cds.cern.ch:1139231. 43rd Rencontres de Moriond on QCD and High Energy Interactions, 2009.

[4] P. Skands. http://home.fnal.gov/ ${ }^{\sim}$ skands/leshouches-plots/.

[5] T. Sjöstrand and P. Skands. Multiple Interactions and the Structure of Beam Remnants. Journal of High Energy Physics, 3:53-+, March 2004.

[6] M. Britsch, N. Gagunashvili, and M. Schmelling, 2008. Talk at the ACAT2008 Workshop: PoS(ACAT08)086. 